\title{
Erratum to: Helicobacter pylori Isolated from a Patient with Ménétrier's Disease Increases Hepatocyte Growth Factor mRNA Expression in Gastric Fibroblasts: Comparison with Helicobacter pylori Isolated from Other Gastric Diseases
}

\section{Takeshi Ishikawa - Takashi Ando - Hiroshi Obayashi - Nami Nakabe • Mika Okita • Yutaka Isozaki - Yasuyuki Nagao · Hirokazu Oyamada • Yoshihiro Nakajima $\cdot$ Haruki Kato $\cdot$ Satoshi Kokura $\cdot$ Yuji Naito • \\ Norimasa Yoshida $\cdot$ Toshikazu Yoshikawa}

Published online: 31 March 2012

(C) Springer Science+Business Media, LLC 2012

Erratum to: Dig Dis Sci (2008) 53:1785-1791

DOI 10.1007/s10620-007-0070-4

We noticed errors in Figs. 3 and 4 and in their respective captions of the published article. The correct figures and captions are given below:

The online version of the original article can be found under doi:10.1007/s10620-007-0070-4.

T. Ishikawa $(\bowtie) \cdot$ M. Okita · Y. Isozaki · Y. Nagao ·

H. Oyamada

Department of Gastroenterology, Matsushita Memorial Hospital,

5-55 Sotojima-cho, Moriguchi City, Osaka 570-8540, Japan

e-mail: iskwt@koto.kpum.ac.jp

T. Ando $\cdot$ N. Nakabe $\cdot$ H. Kato $\cdot$ S. Kokura $\cdot$ Y. Naito ·

T. Yoshikawa

Inflammation and Immunology, Graduate School of Medical

Science, Kyoto Prefectural University of Medicine, Kyoto, Japan

H. Obayashi

Institute of Bio-Response Informatics, Kyoto, Japan

Y. Nakajima

Department of Central Laboratory, Matsushita Memorial

Hospital, Moriguchi City, Japan

N. Yoshida

Molecular Gastroenterology and Hepatology, Graduate School of Medical Science, Kyoto Prefectural University of Medicine, Kyoto, Japan 
(A)

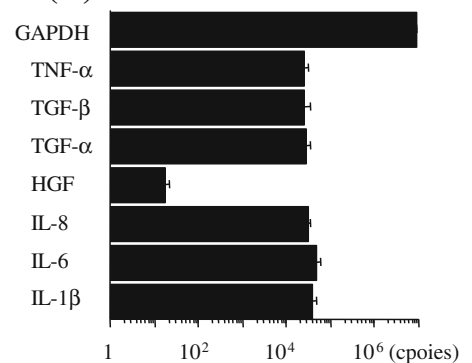

(E)

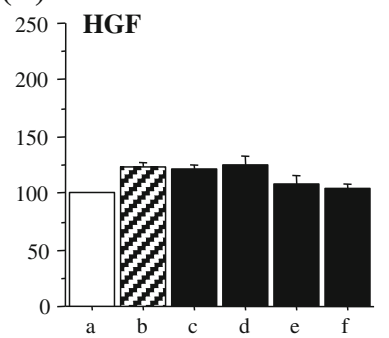

(B)

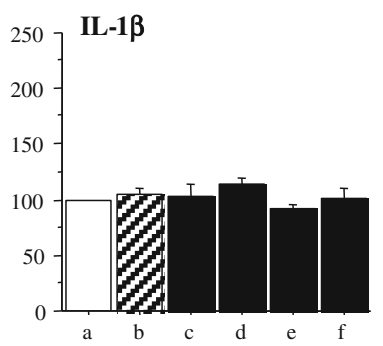

(F)

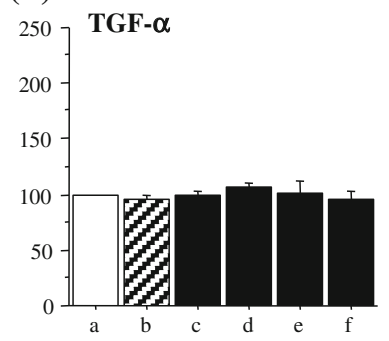

(C)

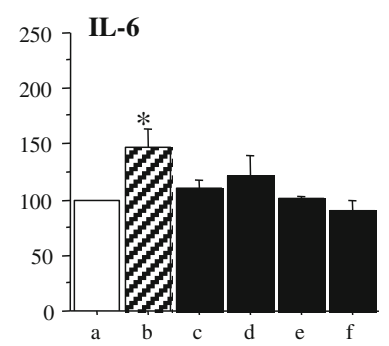

(G)

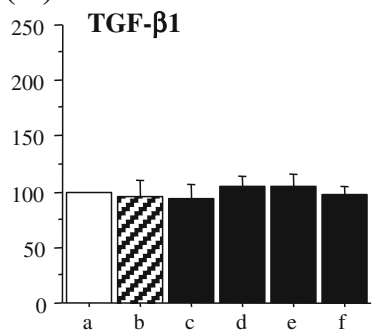

(D)

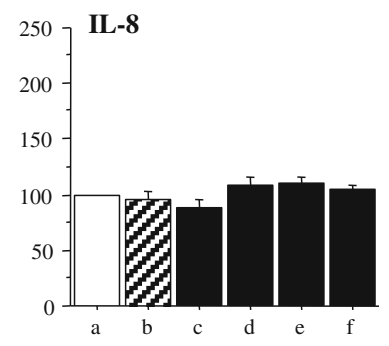

(H)

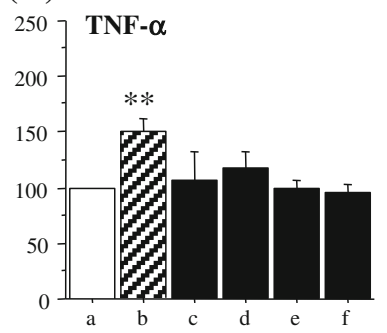

Fig. 3 a Quantitative RT-PCR of GADPH and cytokine mRNA expression induced by $H$. pylori ATCC43504 (control); b relative mRNA expression of IL- $1 \beta$, $\mathbf{c}$ IL-6, $\mathbf{d}$ IL-8, e HGF, $\mathbf{f}$ TGF- $\alpha$, g TGF$\beta 1$, and $\mathbf{h}$ TNF- $\alpha$ induced by different $H$. pylori strains in MKN45 cells. H. pylori strains: (a) ATCC43504, (b) MH1401, (c) MH1208,

(A)

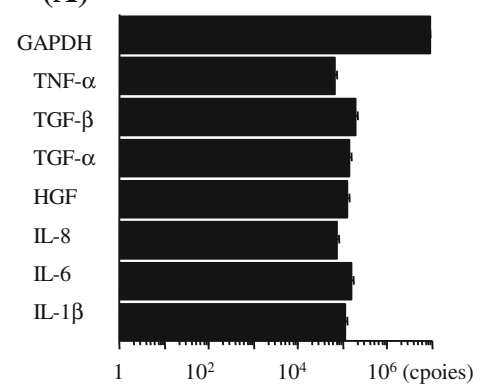

(E)

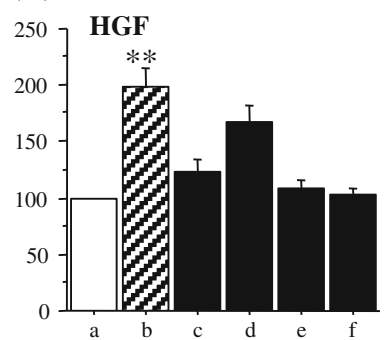

(B)

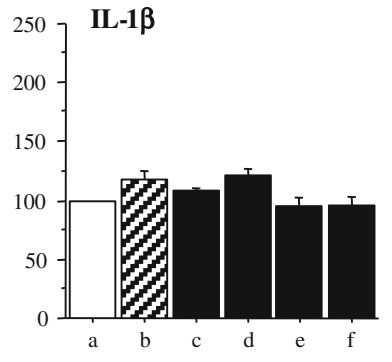

(F)

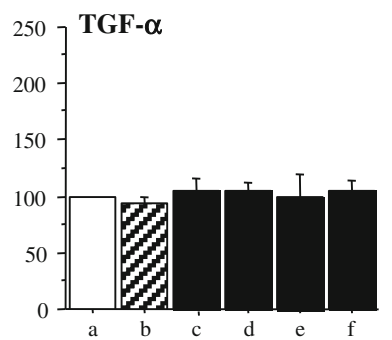

(C)

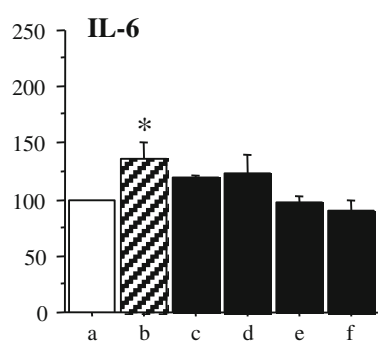

(G)

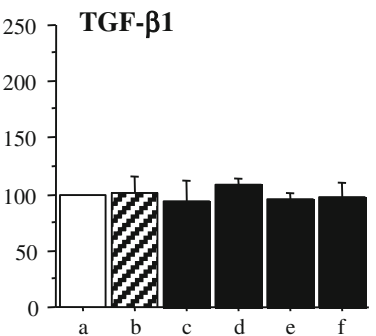

(D)

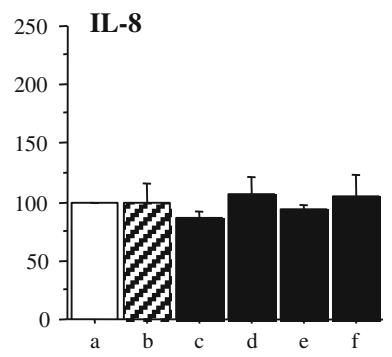

(H)

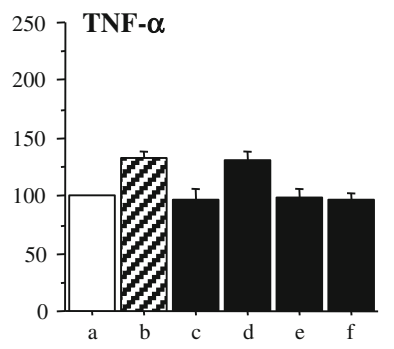

Fig. 4 a Quantitative RT-PCR of GADPH and cytokine mRNA expression induced by $H$. pylori ATCC43504 (control); b relative mRNA expression of IL- $1 \beta$, $\mathbf{c}$ IL-6, $\mathbf{d}$ IL-8, e HGF, $\mathbf{f}$ TGF- $\alpha$, $\mathbf{g}$ TGF$\beta 1$, and $\mathbf{h}$ TNF- $\alpha$ induced by different $H$. pylori strains in human gastric fibroblasts. H. pylori strains: (a) ATCC43504, (b) MH1401, (c) MH1208, (d) MH1303, (e) MH1112, and (f) MH1115. mRNA

expression was standardized against GAPDH mRNA quantification, and is expressed relative to the control $H$. pylori strain $(=100 \%)$. Results are expressed as the mean $\pm \mathrm{SD}$ of three independent experiments. $* p<0.05(b)$ versus $(e)$ or $(f)$. $* * p<0.001(b)$ versus $(a),(c),(e)$ or $(f)$ 